\title{
Estudo de validade da escala de sobrecarga de familiares cuidadores de pacientes psiquiátricos
}

\author{
Burden of care in relatives of psychiatric patients: Validity \\ study of the Family Burden Interview Scale \\ Marina Bandeira, ${ }^{1,2,3,4,5}$, Maria Glaucia Pires Calzavara ${ }^{1,5,6}$, Ildevane Castro ${ }^{1}$
}

\section{RESUMO}

A sobrecarga de familiares cuidadores de pacientes psiquiátricos tem sido amplamente estudada por pesquisas internacionais, usando escalas validadas, mas raramente no Brasil, talvez devido à carência de escalas validadas deste construto. Objetivo: Esta pesquisa avaliou a validade da versão brasileira da escala Family Burden Interview Schedule (FBIS-BR). Método: Participaram cem familiares de pacientes psiquiátricos de três instituições psiquiátricas de Minas Gerais, entrevistados com aplicação de um questionário sociodemográfico e três escalas de medida: FBIS-BR, BI e SRQ-20. Resultados: A validade de critério da escala FBIS-BR foi analisada pela sua correlação com a escala BI, já validada para o Brasil e que avalia o mesmo construto, obtendo-se correlações significativas, entre 0,23 e 0,69 ( $p<0,01)$.

\section{Palavras-chave}

Família, fardos

relativos à doença,

pacientes psiquiátricos, desinstitucionalização, escala de medida, validade de escalas de medida.
A validade de construto da escala FBIS-BR foi analisada pela sua correlação com a SRQ-20, que avalia um construto diferente, de transtornos psicológicos, porém teoricamente relacionado ao de sobrecarga, tendo-se obtido correlações significativas $(p<0,01)$ entre 0,31 e 0,49 . Conclusão: A escala FBIS-BR constitui um instrumento de medida válido para avaliar a sobrecarga dos familiares cuidadores de pacientes psiquiátricos, podendo ser utilizada em serviços de saúde mental para identificar as dificuldades e as necessidades destes familiares e para direcionar novas intervenções de orientação e suporte.

\section{ABSTRACT}

Family caregivers burden has been frequently reported in international researches using validated scales, but rarely in Brazil, perhaps due to the restrict availability of burden scales. Objective: The present study examined the validity of the Brazilian version of the Family Burden Interview Schedule (FBIS-BR). Method: A sample of 100 family caregivers of psychiatric patients attending three public outpatient services were interviewed with the application of three scales: FBIS-BR, BI and SRQ-20. Socio-demographic data were collected with a standardized form. Results: Criterion validity of the FBIS-BR scale was investigated analyzing its correlation with the BI scale,

1 Universidade Federal de São João del Rei (UFSJ).

2 Conselho Nacional de Desenvolvimento Científico e Tecnológico (CNPq).

3 Université de Montreal.

4 McGill University.

5 Laboratório de Pesquisa em Saúde Mental da UFSJ (Lapsam).

6 Universidade Federal de Minas Gerais (UFMG)

Endereço para correspondência:Marina Bandeira

Rua Antonia da Encarnação Xavier, 120 - 36325-000 - Tiradentes, MG

E-mail: bandeira@ufsj.edu.br 


\section{Keywords}

Scale, family, burden of care, psychiatric patients, deinstitucionalization, scale validity. which evaluates the same construct of family burden and the significant scores obtained varied between 0.23 e $0.69(p<0.01)$. The construct validity of the FBIS-BR scale was evaluated analyzing its correlation with the SRQ-20 scale, which evaluates a different but related construct of psychological disturbance, and the significant scores obtained varied between 0.31 e 0.49. Conclusion: The FBIS-BR scale has good criterion and construct validity indicators to evaluate family burden of caregivers of psychiatric patients. This scale therefore can be used in mental health services, to evaluate the difficulties and needs presented by the family and to elaborated new support and orientation interventions.

\section{INTRODUÇÃO}

A avaliação da sobrecarga dos familiares cuidadores de pacientes psiquiátricos se tornou necessária, no contexto da desinstitucionalização psiquiátrica, tendo em vista o maior envolvimento das famílias nos cuidados cotidianos aos pacientes. Avaliar o impacto do papel de cuidador nas famílias pode fornecer informações importantes para o desenvolvimento de intervenções psicossociais e educativas capazes de ajudar efetivamente essas famílias e melhorar a qualidade do atendimento nos serviços de saúde mental. O sucesso da reinserção social dos pacientes psiquiátricos só poderá ser plenamente alcançado se os familiares também forem atendidos em suas dificuldades e necessidades, por meio de intervenções que forneçam apoio, orientação e informação. A avaliação da sobrecarga familiar permitirá identificar essas necessidades ${ }^{1-3}$.

Pesquisas internacionais constataram que os familiares se sentem sobrecarregados no papel de cuidadores, embora possam também sentir satisfação por cuidar do paciente ${ }^{4}$. Estudos de revisão de literatura da área $a^{5-7}$ apontaram que a sobrecarga dos familiares resulta das tarefas advindas do papel de cuidador e das mudanças que ocorrem em sua vida social e profissional, pois eles precisam colocar as necessidades dos pacientes como prioridade, deixando suas próprias necessidades em segundo lugar. Além disso, esses estudos mostraram que a sobrecarga é agravada pela falta de informação a respeito da doença do paciente, do tratamento utilizado e das estratégias mais adequadas para lidar com os comportamentos problemáticos dos pacientes e para o manejo das situações de crise. A sobrecarga contínua pode resultar comprometimento da saúde física e psicológica dos familiares, com o desenvolvimento de transtornos psicológicos, tais como ansiedade e depressão, provocando conseqüências negativas, tanto no contexto familiar quanto social e do trabalho?. Uma revisão detalhada dos estudos realizados para avaliar as características da sobrecarga familiar e dos fatores associados a um maior grau de sobrecarga encontra-se descrita em Bandeira e Barroso ${ }^{8}$.
Uma avaliação completa da sobrecarga requer o uso de um instrumento de medida multidimensional, tendo em vista a natureza deste construto. O conceito de sobrecarga envolve dois aspectos, as sobrecargas objetiva e a subjetiva, que afetam diversos domínios da vida dos familiares ${ }^{4}$. A sobrecarga objetiva se refere ao desempenho das tarefas de assistência ao paciente e da supervisão dos seus comportamentos problemáticos, assim como os transtornos e as restrições que ocorrem na vida social e ocupacional dos familiares e o impacto financeiro. A sobrecarga subjetiva se refere às percepções e sentimentos dos familiares, tais como suas preocupações com o paciente, a sensação de peso a carregar e de incômodo ao exercer algumas das funções do papel de cuidador ${ }^{5-7}$. Um instrumento de medida multidimensional permite identificar os domínios mais afetados da vida dos familiares em subescalas que fornecem escores independentemente do grau de sobrecarga.

$\mathrm{Na}$ literatura internacional, são encontradas pesquisas que avaliaram a sobrecarga familiar com instrumentos de medida validados para identificar quais domínios da vida dos familiares são mais afetados e os fatores associados à sobrecarga. No Brasil, embora haja interesse pelo estudo de famílias de pacientes psiquiátricos ${ }^{9,10}$, ainda é escasso o número de publicações, em periódicos científicos, de pesquisas que avaliem, especificamente, a sobrecarga desses familiares, utilizando escalas de medida ${ }^{11-13}$. A escassez dessas pesquisas pode estar relacionada à carência de instrumentos de medida validados para o Brasil, para avaliar a sobrecarga dos familiares cuidadores de pacientes psiquiátricos. A validação de instrumentos de medida é importante para garantir dados mais seguros nas pesquisas desta temática e também para possibilitar a comparabilidade dos resultados obtidos em diferentes pesquisas ${ }^{14}$.

O objetivo da presente pesquisa foi aprofundar o estudo das propriedades psicométricas de uma escala de medida da sobrecarga familiar, a Family Burden Interview Schedule (FBIS), que consiste de uma escala multidimensional, portanto, adequada para avaliar o conceito multifacetado de sobrecarga. Essa escala, elaborada por Tessler and Gamache ${ }^{4}$ já foi submetida ao processo de adaptação transcultural para o Brasil, com a sigla FBIS-BR ${ }^{15}$ e foi submetida ao estudo 
de sua confiabilidade ${ }^{16}$. No presente trabalho, foi avaliada a validade de construto e critério dessa escala.

\section{MÉTODO}

\section{Participantes}

Participaram desta pesquisa cem familiares de pacientes psiquiátricos de três instituições psiquiátricas públicas: os Centros de Atenção Psicossocial (CAPS) das cidades de São João del Rei e de Lavras e a Fundação Hospitalar do Estado de Minas Gerais (FHEMIG) da cidade de Barbacena. Os familiares constituíam os principais cuidadores dos pacientes, residiam com os eles e foram selecionados aleatoriamente entre os que freqüentavam os serviços. Foram excluídos os familiares acima de 80 anos e os que apresentavam diagnóstico psiquiátrico.

A amostra foi composta por familiares de ambos os sexos, 20 do sexo masculino e 80 do sexo feminino; com idade média de 51,04 anos, a idade mínima de 18 anos e a máxima de 79 anos. Considerando o grau de parentesco, a maioria da amostra (38\%) se constituía de mães ou pais dos pacientes e, em segundo lugar, de irmãos (23\%), os demais cônjuges (13\%), filhos (11\%) ou outro tipo de parentesco (14\%). Com relação ao nível socioeconômico das famílias, com quem os pacientes moravam, a maioria tinha renda total de um a três salários-mínimos (67\%), enquanto 15\% das famílias recebiam de 3,1 a quatro salários-mínimos, 17\% recebiam mais de quatro salários-mínimos e apenas 1\% recebia menos de um salário-mínimo.

Esses familiares cuidavam de pacientes psiquiátricos cujos diagnósticos, em sua maioria, consistiam de transtornos do espectro esquizofrênico (68\%), sendo 55\% com diagnóstico de esquizofrenia, $8 \%$ de transtorno esquizotípico e 5\% de transtorno esquizoafetivo. A duração média da doença psiquiátrica era de 17 a 20 anos. Os pacientes eram de ambos os sexos, $46 \%$ do sexo masculino e $54 \%$ do sexo feminino. A idade média era de 40 a 48 anos, a idade mínima 12 e a máxima 74 . 0 número médio de crises ocorridas no último ano foi de 4,3, e o número médio de internações ocorridas durante toda a vida era de 5,8. Os pacientes apresentaram, em média, 6,23 comportamentos problemáticos, descritos por seus familiares.

\section{Instrumentos de medida}

\section{Escala de avaliação da sobrecarga familiar FBIS-BR}

Esta escala, elaborada por Tessler e Gamache ${ }^{4}$, avalia cinco dimensões da sobrecarga dos familiares de pacientes psiquiátricos. As dimensões avaliadas são: a) assistência na vida cotidiana do paciente; b) supervisão aos comportamentos problemáticos do paciente; c) gastos financeiros do familiar com o paciente; d) impacto nas rotinas diárias da família; e e) preocupações do familiar com o paciente. As questões da escala se referem aos últimos 30 dias.

A escala avalia as sobrecargas objetiva e subjetiva. A sobrecarga objetiva é avaliada por meio da freqüência de assistências e supervisões do familiar no cuidado cotidiano com o paciente e a freqüência de alterações na rotina de sua vida, com as seguintes alternativas de resposta: $1=$ nenhuma vez, 2 = menos que uma vez por semana, 3 = uma ou duas vezes por semana, $4=$ de três a seis vezes por semana e $5=$ todos os dias. A sobrecarga subjetiva é avaliada por meio do grau de incômodo sentido pelo familiar ao exercer o papel de cuidador e das suas preocupações com o paciente. Para a avaliação do grau de incômodo, as opções de resposta são: $1=$ nem um pouco, 2 = muito pouco, $3=$ um pouco e 4 = muito. Para a avaliação das preocupações, as alternativas de resposta são: 1 = nunca, 2 = raramente, 3 = às vezes, $4=$ freqüentemente e $5=$ sempre ou quase sempre.

A primeira dimensão da escala (Subescala A) avalia as sobrecargas objetivas e subjetivas, por meio respectivamente de nove questões que avaliam a freqüência da assistência prestada pelo familiar ao paciente e de nove questões que avaliam o grau de incômodo sentido ao prestar essa assistência.

A segunda dimensão (Subescala B) também avalia as sobrecargas objetivas e subjetivas, por meio respectivamente de oito questões que avaliam a freqüência com que o familiar teve que supervisionar os comportamentos problemáticos do paciente e de oito questões que avaliam grau de incômodo sentido pelo familiar ao lidar com esses comportamentos.

A terceira dimensão (Subescala C) avalia a sobrecarga financeira e é composta por cinco questões, que não entram no cálculo dos escores de sobrecarga, embora forneçam informações adicionais. A primeira questão dicotômica avalia se o familiar cobriu alguma despesa do paciente sem receber o dinheiro gasto de volta, a segunda questão avalia os gastos que o paciente teve com 14 itens de despesas, tais como transporte, alimentação, medicamentos, moradia, plano de saúde, entre outras. A terceira e a quarta questões são abertas e avaliam a contribuição do paciente para cobrir suas próprias despesas e a quantia gasta pela família com as despesas do paciente. A última questão avalia a freqüência com que o familiar sentiu pesados os gastos com o paciente para o orçamento familiar.

A quarta dimensão (Subescala D) avalia a sobrecarga objetiva, por meio de quatro questões que avaliam o impacto do papel do cuidador na vida do familiar, em termos da freqüência das alterações ocorridas na rotina familiar, nos últimos 30 dias. Além disso, uma quinta questão, que não entra no cálculo dos escores desta subescala avalia, de forma global, em que grau o cuidador teve mudanças permanentes na sua vida social e ocupacional e uma última questão avalia quais foram essas mudanças ocorridas em seis aspectos da 
vida do cuidador. A quinta dimensão da escala (Subescala E) é constituída por sete questões que avaliam a sobrecarga subjetiva do cuidador, em termos da freqüência das suas preocupações com o paciente.

A escala FBIS-BR foi traduzida e adaptada para o Brasil por Bandeira, Calzavara e Varella ${ }^{15}$, tendo seguido os procedimentos recomendados pela literatura internacional para a adaptação transcultural de instrumentos de medida ${ }^{17-19}$, incluindo tradução e retradução, análise por uma comissão de especialistas e estudo-piloto com a população-alvo. A escala FBIS-BR foi também submetida ao estudo de sua confiabilidade por Bandeira, Calzavara, Freitas e Barroso ${ }^{16}$, tendo apresentado, na análise de sua consistência interna, valores de alfa de Cronbach de 0,82 para o escore global de sobrecarga objetiva, 0,92 para a escala global subjetiva e valores de alfa entre 0,58 a 0,90 para as subescalas. 0 estudo da estabilidade temporal da escala apresentou escores significativos de correlação de Pearson entre as aplicações do teste e do reteste, variando de 0,54 a 0,90 para as subescalas e o escore global.

Uma descrição detalhada dessa escala, com instruções de aplicação e formas de correção para cálculo dos escores de sobrecarga, encontra-se acessível no site do Laboratório de Pesquisa em Saúde Mental da UFSJ (www.lapsam.ufs. edu.br).

\section{Escala Burden Interview (BI)}

Esta escala, elaborada por Zarit ${ }^{20}$ e validada para o Brasil por Taub, Andreoli e Bertolucci ${ }^{21}$, possui 22 itens que avaliam a sobrecarga subjetiva dos familiares cuidadores de pacientes psiquiátricos, em termos da expressão de seus sentimentos referentes ao papel de cuidador. A escala avalia o impacto da sobrecarga nos seguintes aspectos da vida do familiar: saúde, vida social e pessoal, situação financeira, bem-estar emocional e relações interpessoais. As alternativas de resposta para cada item estão apresentadas em uma escala de 0 a 4 , na qual $0=$ nunca; $1=$ raramente; 2 = algumas vezes; 3 = freqüentemente e 4 = sempre.

O último item da escala avalia, de forma global, quanto o familiar se sente sobrecarregado por cuidar de um paciente psiquiátrico. Para esse item, as alternativas de resposta são: 0 = nem um pouco, $1=$ um pouco, 2 = moderadamente, 3 = muito, 4 = extremamente.

\section{Escala Self Reporting Questionnaire (SRQ-20)}

Esta escala foi desenvolvida por Harding et al. ${ }^{22}$ e validada para o Brasil por Mari e Williams ${ }^{23}$. Esse instrumento é utilizado para identificar transtornos mentais comuns, definidos por Goldberg e Huxley ${ }^{24}$ como "transtornos comumente encontrados na população e que sinalizam uma interrupção do funcionamento normal". Trata-se de sintomas como insônia, fadiga, irritabilidade, esquecimento, dificuldade de concentração e queixas somáticas ${ }^{25}$. A escala é composta por 20 questões dicotômicas que avaliam a presença de sintomas físicos (quatro questões) e de distúrbios psicoemocionais (16 questões). Os resultados são analisados a partir da soma dos itens. $O$ ponto de corte fixado a partir de entrevistas psiquiátricas no estudo original foi de $7 / 8^{23}$, enquanto outras análises posteriores por meio da técnica de Relative Operating Characteristic (ROC) analysis, segundo Ludermir $^{25}$, apontam um ponto de corte de 5/6 de maior sensibilidade e especificidade.

\section{Questionário sociodemográfico e clínico}

Foi elaborado um questionário para a identificação das características sociodemográficas do paciente e do familiar, bem como as variáveis clínicas do paciente. As características sociodemográficas coletadas foram: idade, sexo, estado civil, escolaridade e renda. As variáveis clínicas coletadas se referem ao tipo de diagnóstico do paciente e informações sobre a doença (duração, número de internações e de comportamentos problemáticos) apresentada pelo paciente, o tipo de tratamento e as medicações utilizadas.

\section{Procedimento}

Depois da aprovação do projeto pelas diretorias dos serviços de saúde mental participantes e pela comissão de avaliação de pesquisas da UFSJ, que avalia a pertinência, a relevância e o respeito à ética, os familiares foram contatados a partir dos prontuários dos serviços para agendamento das entrevistas. As entrevistas eram realizadas individualmente, nos serviços onde os pacientes recebiam tratamento e raramente em casa, dependendo da disponibilidade dos familiares. Os entrevistadores eram estagiários do curso de Psicologia, previamente treinados pelos pesquisadores, a fim de se obter uma padronização do procedimento de aplicação da escala, embora a escala não necessite de uma formação clínica especial para ser aplicada. Os indivíduos eram informados sobre os objetivos da pesquisa e sobre o sigilo dos dados e assinavam um termo de consentimento informado. Durante a entrevista, as questões eram lidas pelo entrevistador, e as respostas dos indivíduos, anotadas. Para que houvesse melhor entendimento do respondente, foram utilizados cartões contendo as alternativas de resposta de cada pergunta. Depois da entrevista, era fornecido ao familiar o número de telefone para contato com a equipe de pesquisa, caso o familiar tivesse alguma dúvida sobre a pesquisa ou quisesse saber os resultados.

\section{Análise dos dados}

A análise da validade da escala FBIS-BR abrangeu dois procedimentos de validação descritos por Pasquali26. 0 primeiro procedimento corresponde à validade concorrente, avaliada por meio da correlação de Pearson entre os escores obtidos na escala FBIS-BR e na escala BI, a qual avalia o mesmo construto de sobrecarga familiar. Essa validade consiste 
de um subtipo da validade de critério, que diz respeito à eficácia de um teste em medir um construto que se relacione com um desempenho específico do indivíduo, o qual é considerado como critério. Quando este critério se refere a um desempenho atual e a coleta de dados é feita simultaneamente à aplicação do teste, denomina-se validade concorrente ${ }^{26}$. Segundo Pasquali, testes já validados podem ser utilizados como critério para a validação de outros testes, quando ambos se relacionam ao mesmo construto.

O segundo procedimento envolveu a análise da validade de construto da escala FBIS-BR, por meio da técnica da validação convergente, que consiste da análise da hipótese de que o teste deva correlacionar-se significativamente com outras medidas de construtos diferentes, porém teoricamente relacionadas ao construto medido ${ }^{26}$. Foi analisada a validade convergente da FBIS-BR, por meio da correlação de Pearson com a escala SRQ-20, que avalia um construto diferente porém relacionado à sobrecarga familiar, segundo a literatura da área. A escala SRQ-20 avalia o construto denominado distress, "desconforto emocional" ou transtornos mentais comuns (TMC), que pode resultar da sobrecarga sofrida pelos cuidadores. Portanto, o FBIS-BR deveria correlacionar-se com a escala SRQ-20, segundo esta análise de hipótese.

\section{RESULTADOS}

Na tabela 1 encontram-se os resultados da análise estatística descritiva e das análises correlacionais. Estão apresentadas as médias e os desvios-padrão dos itens da escala, além dos valores dos coeficientes de correlação de Pearson entre os escores das subescalas da FBIS-BR e as escalas BI e SRQ-20.

\section{Análise descritiva dos resultados}

A tabela 1 apresenta os resultados descritivos das sobrecargas objetiva e subjetiva apresentadas pelos familiares. No que se refere à sobrecarga objetiva, pode-se observar, na tabela 1, que o escore global foi de 2,29 e que os es- cores médios obtidos nas subescalas variaram entre 1,68 e 2,72. A subescala que apresentou o escore médio mais elevado de sobrecarga objetiva foi a que avalia a assistência ao paciente na vida cotidiana. Esses dados indicam que houve freqüência desse tipo de assistência que se situou, em média, entre menos de uma vez e uma a duas vezes por semana. A subescala que apresentou o escore médio menos elevado de sobrecarga objetiva foi a D, que se refere às alterações ocorridas na rotina social e profissional do familiar, em decorrência de sua condição de cuidador. Isso indica que houve baixa freqüência desse tipo de alterações, entre nenhuma vez e menos de uma vez por semana, em média.

Quanto à sobrecarga subjetiva, pode-se observar, na tabela 1, que o escore global foi de 2,89 e os escores médios variaram entre 1,77 e 3,49. A subescala que apresentou o escore médio mais elevado de sobrecarga subjetiva foi a das preocupações do familiar com o paciente (E), que ocorreram com freqüência média entre às vezes e freqüentemente. A subescala que apresentou o menor escore médio de sobrecarga subjetiva foi a da assistência ao paciente na vida cotidiana. O grau de incomodo sentido pelos familiares ao fazer essas tarefas foi de 1,77, o qual se situa entre nem um pouco e muito pouco.

Dados mais detalhados sobre as sobrecargas objetiva e subjetiva em cada um dos itens da escala podem ser encontrados em Barroso, Bandeira e Nascimento ${ }^{13}$.

\section{Validade de critério: análise correlacional entre a escala FBIS-BR e a BI}

Os resultados referentes à validade de critério da escala FBIS-BR, analisados em termos da sua correlação com a escala Bl, encontram-se na tabela 1. Pode-se observar que foram obtidas correlações significativas entre a escala BI e os escores obtidos para todas as subescalas da FBIS-BR $(p<0,01)$ e escores globais. Os coeficientes de correlação variaram entre 0,23 e 0,69. A subescala que apresentou o coeficiente de correlação mais elevado foi a que se refere às alterações ocorridas na rotina da vida social e profissional do familiar em decorrência de seu papel de cuidador (D).

Tabela 1. Médias, desvios-padrão dos escores das subescalas da FBIS-BR e coeficientes de correlação de Pearson entre os escores das escalas FBIS-BR, BI e SRQ-20.

\begin{tabular}{|c|c|c|c|c|c|}
\hline Subescalas & Dimensões da sobrecarga & Médias & Desvios-padrão & Correlaçöes com BI & Correlações com SRQ-20 \\
\hline \multirow[t]{2}{*}{ A - Assistência na vida cotidiana } & Objetiva & 2,72 & 0,95 & $0,45^{*}$ & $0,32^{*}$ \\
\hline & Subjetiva & 1,77 & 0,75 & $0,38^{*}$ & $0,36^{*}$ \\
\hline \multirow{2}{*}{ B - Supervisão aos comportamentos problemáticos } & Objetiva & 2,00 & 0,89 & $0,57^{*}$ & $0,31^{*}$ \\
\hline & Subjetiva & 3,16 & 0,86 & $0,23^{*}$ & 0,11 \\
\hline D - Impacto na rotina & Objetiva & 1,68 & 0,97 & $0,69^{*}$ & $0,36^{*}$ \\
\hline E - Preocupações com o paciente & Subjetiva & 3,49 & 0,89 & $0,43^{*}$ & $0,49^{*}$ \\
\hline Escore global objetivo & & 2,29 & 0,68 & $0,67^{*}$ & $0,38^{*}$ \\
\hline Escore global subjetivo & & 2,89 & 0,69 & $0,47^{*}$ & $0,52^{*}$ \\
\hline
\end{tabular}

* $p<0,01$

Nota: a subescala não foi analisada por conter dados financeiros. 
A subescala que apresentou o menor coeficiente de correlação foi a que se refere às supervisões aos comportamentos problemáticos do paciente (B).

\section{Validade de construto: análise correlacional entre a escala FBIS-BR e a SRQ-20}

Com relação à análise correlacional com a escala SRQ-20, os resultados mostraram correlações significativas entre $O$ escore global dessa escala e os escores globais da FBIS-BR e com a maioria das subescalas da FBIS-BR ( $p<0,01)$. Os coeficientes de correlação que foram significativos variaram entre 0,31 e 0,52. A subescala que apresentou o coeficiente de correlação mais elevado foi a $E$, que se refere às preocupações do familiar com o paciente. A subescala que apresentou o menor coeficiente de correlação foi a que se refere às supervisões aos comportamentos problemáticos do paciente (B). Apenas a dimensão subjetiva dessa subescala não apresentou correlação significativa com a escala SRQ-20, o que talvez se possa explicar pela presença de dados faltosos, uma vez que nem todos os comportamentos problemáticos ocorrem com todos os pacientes.

\section{DISCUSSÃO}

Os resultados obtidos apontam a validade de critério da escala FBIS-BR, tendo em vista que ela apresentou correlação significativa com o critério adotado de avaliação da sobrecarga familiar, que foi a escala Bl, já validada anteriormente para o Brasil e que avalia o mesmo construto de sobrecarga familiar. As correlações, embora significativas, foram de intensidades moderadas, o que talvez se explique pelas diferenças que existem entre as duas escalas, tais como o número de itens, a forma de redação dos itens, o número e o tipo de alternativas de resposta e o conteúdo das questões, que eram mais diversificadas e abrangentes na FBIS-BR, enfocando tanto aspectos objetivos quanto subjetivos da sobrecarga. Diferenças nos valores das correlações obtidas para as subescalas podem talvez se explicar pela diferença observada na ocorrência de dados faltosos, principalmente nas subescalas subjetivas, o que resulta diferentes números de indivíduos incluídos nas análises estatísticas das subescalas. A correlação mais elevada obtida para a subescala referente a alterações na vida social e profissional do cuidador talvez se explique pelo fato de o conteúdo das questões da escala BI se assemelhar mais com o desta subescala.

Os resultados do presente trabalho demonstraram ainda a validade de construto da escala FBIS-BR, avaliada pelo teste de hipótese, segundo o qual um instrumento de medida possui validade de construto quando ele correlaciona significativamente com outras medidas que avaliam construtos diferentes, porém com as quais ele esteja teoricamente relacionado ${ }^{26}$. Tendo em vista a diferença entre os construtos avaliados, espera-se, portanto, uma correlação moderada. O construto distress ou desconforto emocional indica a presença de sintomas clínicos que sinalizam uma interrupção do funcionamento normal, também denominado transtornos mentais comuns ou TMC ${ }^{24}$. Trata-se de um construto diferente do de sobrecarga e que tem sido avaliado pela escala SRQ-20. Diferenças entre sobrecarga e desconforto emocional também foram observadas em outros estudos ${ }^{12}$. Entretanto, esses dois contrutos são teoricamente relacionados, pois se considera que a sobrecarga sofrida pelos familiares que cuidam de pacientes psiquiátricos pode afetar seu estado psicológico, resultando desconforto emocional e o desenvolvimento de transtornos mentais comuns. A correlação significativa obtida entre esses dois instrumentos, no presente trabalho, confirmando a hipótese postulada, confirmam, portanto, a validade de construto da escala FBIS-BR.

Comparando-se os dois tipos de análises descritas anteriormente, pode-se observar, em geral, que as correlações obtidas com a escala Bl foram mais elevadas do que as observadas para a escala SRQ-20. Estes resultados constituem indicadores da validade multitraço da escala FBIS-BR. Segundo Contandriopoulos et al. ${ }^{27}$, uma escala possui validade multitraço quando possui correlações mais elevadas com uma outra escala que meça o mesmo construto do que com uma escala que meça um construto diferente, este tipo de validade, constituindo-se, segundo o autor, uma das variantes da validade de construto.

\section{CONCLUSÃO}

A partir dos resultados obtidos, pode-se concluir que a escala FBIS-BR possui propriedades psicométricas adequadas de validade de critério e de construto, constituindo-se um instrumento de medida válido para avaliar as sobrecargas objetiva e subjetiva, em diversos domínios da vida dos familiares cuidadores de pacientes psiquiátricos. Por ser uma escala multidimensional, poderá avaliar de forma mais completa e diferenciada esse construto por meio de suas subescalas. Instrumentos de medida validados são importantes no contexto da pesquisa em saúde mental, pois eles permitem a obtenção de dados mais seguros e possibilitam a comparabilidade dos resultados de diferentes estudos ${ }^{14}$.

Essa escala pode ser utilizada, portanto, em serviços de saúde mental, visando a conhecer as principais dificuldades apresentadas pelos familiares no seu papel de cuidadores. O uso da escala permitirá identificar, de modo mais preciso, as dimensões da vida dos familiares que foram mais afetadas, assim como as suas necessidades de suporte e orientação por parte dos profissionais de saúde mental. 
Pesquisas futuras poderão aprofundar o estudo da validade de construto da escala FBIS-BR, verificando a capacidade desta escala em discriminar grupos distintos de cuidadores para os quais se espera teoricamente que devam diferir em seu grau de sobrecarga. Por exemplo, podem ser avaliados cuidadores que morem ou não com o paciente ou grupos com diferentes graus de parentesco com o paciente ou que cuidam de pacientes com diagnósticos diferentes.

Conflito de interesses: Este artigo faz parte de um projeto de pesquisa financiado pela FAPEMIG e pelo CNPq.

\section{REFERÊNCIAS}

1. St Onge M, Lavoie F, Cormier H. Les difficultés perçues para des mères de personnes atteintes de troubles psychotiques face au système de soins professionnels. Sante Ment Que. 1995;20(1):89-118.

2. Hanson JG, Rapp CA. Families' perceptions of community mental health programs for their relatives with a severe mental illness. Community Ment Health J. 1992;28(3):181-97.

3. Solomon P, Beck S, Gordon B. Family members' perspectives on psychiatric hospitalization and a discharge. Community Ment Health J. 1988;24(2):108-17.

4. Tessler RC, Gamache GM. The Family Burden Interview Schedule - Short Form (FBIS/SF). In: Sederer L, Dickey B (eds). Outcome Assessment in Clinical Practice. Baltimore: Williams \& Williams, 1996. p.110-2.

5. Maurin JT, Boyd CB. Burden of mental illness on the family: a critical review. Arch Psychiatr Nurs. 1990;4(2):99-107.

6. Loukissa AD. Family burden in chronic mental illness: a review of research studies. J Adv Nursing. 1995;21:248-55.

7. Rose LE. Families of psychiatric patients: a critical review and future research directions. Arch Psychiatr Nurs. 1996;10(2):67-76.

8. Bandeira M, Barroso S. Sobrecarga das famílias de pacientes psiquiátricos. J Bras de Psiquiatr. 2005;54(1):34-46.

9. Villares (C, Mari JJ. Esquizofrenia e contexto familiar. In: Shirakawa I, Chaves AC, Mari JJ, (eds.). 0 desafio da esquizofrenia. São Paulo: Lemos Editorial, 1998. p. 243-5.

10. Villares CC. Adaptação transcultural de intervenções psicossociais na esquizofrenia. Rev Bras Psiquiatr. 2000;22(1):53-5.

11. Garrido R, Menezes PR. Impacto em cuidadores de idosos com demência atendidos em um serviço psicogeriátrico. Rev Saude Publica. 2004;38(6):835-841.
12. Scazufca M, Menezes PR, Almeida P. Caregiver burden in an elderly population with depression in São Paulo, Brazil. Soc Psychiatry Psychiatr Epidemiol. 2002;37:416-22.

13. Barroso S, Bandeira M, Nascimento E. Sobrecarga de familiares cuidadores de pacientes psiquiátricos atendidos na rede pública. Rev de Psiquiatr Clin. 2007;34(6)270-7.

14. Ruggeri M. Patients' and relatives' satisfaction with psychiatric services: the state of the art of its measurement. Soc Psychiatry Psychiatr Epidemiol. 1994;29:212-27.

15. Bandeira M, Calzavara MGP, Varella AAB. Escala de sobrecarga dos familiares de pacientes psiquiátricos: adaptação transcultural para 0 Brasil (FBIS-BR). J Bras Psiquiatr. 2005;54(3):206-14.

16. Bandeira M, Calzavara MGP, Freitas L, Barroso SM. Family Burden Interview Scale for relatives of psychiatric patients (FBIS-BR): reliability study of the Brazilian version. Rev Bras de Psiquiatr. 2007;29(1)47-50.

17. Vallerand RJ. Vers une méthodologie de validation trans-culturelle de questionnaires psychologiques: implications pour la recherche en langue française. Can Psychol. 1989;30(4):662-80.

18. Guillemin F; Bombardier C, Beaton D. Cross-Cultural adaptation of health-related quality of life measures: literature review and proposed guidelines. J Clin Epidemiol. 1993;46(12):1417-32

19. Jorge MR. Adaptação transcultural de instrumentos de pesquisa em saúde mental. In: Gorestein C, Andrade LHS, Zuardi AW (eds.). Escalas de avaliação clínica em psiquiatria e psicofarmacologia. São Paulo: Lemos Editorial; 2000. p. 53-8.

20. Zarit SH, Reever KE, Bach-Peterson J. Relatives of impaired elderly: correlates of feeling of burden. Gerontologist. 1980;20(6):649-55.

21. Taub A, Andreoli SB, Bertolucci PH. Dementia caregiver burden: reliability of the Brazilian version of the Zarit caregivers burden interview. Cad de Saude Publica. 2004;20(2):372-6.

22. Harding TW; de Arango MV; Baltazar J, Climent CE, Ibrahim HHA, Ladrido-Ignacio L, et al. Mental disorders in primary health care: A study of the frequency and diagnosis in four developing countries. Psychol Med. 1980;10:231-41.

23. Mari JJ, Williams P. A validity study of a psychiatric screening questionnaire (SRQ-20) in primary care in the city of São Paulo. Br J Psychiatry. 1986;148: 23-6.

24. Goldberg D, Huxley P. Common Mental Disorders: A Bio-Social Model. Londres: Tavistock Publications/New York: Routledge, 1992.

25. Ludermir AB. Inserção produtiva, gênero e saúde mental. Cad. Saúde Pública. 2000;16(3):647-59.

26. Pasquali, L. Psicometria: teoria dos testes na psicologia e na educação. Rio de Janeiro: Vozes, 2003.

27. Contandriopoulos AP, Champagne F, Potvin L, Denis JL, Boyle P. Saber preparar uma pesquisa: definição, estrutura, financiamento. São Paulo: Hucitec Abrasco, 1994. 